\title{
COMBINED ANALYSIS OF SOFT AND HARD X-RAY SPECTRA FROM FLARES
}

\author{
A. H. Gabriel, ${ }^{*}$ E. Bely-Dubau, ${ }^{* *}$ J. C. Sherman, ${ }^{*}$ \\ L. E. Orwig ${ }^{* * *}$ and J. Schrijver \\ *Rutherford Appleton Laboratory, Chilton, Oxon, England \\ **Nice Observatory, BP 139, 06003 Nice Cédex. France: \\ **NASA/Goddard Space Flight Center, Greenbelt, MD, U.S.A. \\ +Space Research Laboratory, 3527 HS Utrecht. The Netherlands
}

\section{ABSTRACT}

A method has been developed for interpreting the combined data set from the BCS, HXIS and HXRBS instruments on the SMM. The observations are fitted to a model including thermal and non-thermal electron components. Analysis of the flare of 1980 June 29, 18.22 UT shows the way in which these components vary through the impulsive and gradual phases.

\section{INTRODUCTION}

The Bent Crystal Spectrometer (BCS), the Hard X-ray Imaging Spectrometer (HXIS) and the Hard $\mathrm{X}$-Ray Burst Spectrometer (HXRBS) on the Solar Maximun Mission together cover both line and continuum $x$-ray emission from $4 \mathrm{kV}$ up to $500 \mathrm{kV}$. Varlous methods have been used for interpreting the observations from these data. The methods differ widely in approach, and in the assumptions made for each of the instruments. The results deduced from the analyses are not in general in good agreement, nor are they well determined, taking account of uncertalnties in the accuracy of the data. In the present work, we search for a selfconsistent interpretation of the intensities observed from all three instruments in order to broaden the energy range of the data, and thereby avoid many of the difficulties.

of these three instruments, BCS and HXRBS spatlally integrate data from the entire flare, whilst HXIS gives spatial resolution. To provide consistency, the HXIS data was integrated over area, and all the analysis carrled out on total flare emission. The Flat Crystal Spectrometer (FCS) recording at lower energies was not included owing to the difficulty of quantitatively relating fluxes from the different spatial, wavelength, and time sampling that it provides.

\section{SMM OBSERVATIONS}

The data used from the BCS included parent and satellite lines from Ca XIX, Fe XXV and Fe XXVI. Since certain families of satellite lines behave similarly with regard to temperature dependence, representative behaviour can in fact be obtained from eight selected Iines:-

$$
\begin{array}{ll}
\text { Ca XIX } & w, q, k \\
\text { Fe XXV } & w, q, j \\
\text { Fe XXVI } & r \text { (resonance), s (satellite) }
\end{array}
$$

Early methods of analysing these data employed directly the theory presented by Bely-Dubau et al. $/ 1,2 /$, and Dubau et al. $/ 3 /$ to derive temperatures from satellite to resonance 1 ine ratios $(\mathrm{e} . \mathrm{g} \cdot$ as $\mathrm{k} / \mathrm{w})$ and emission measures from resonance line absolute intensities. In a strict sense such methods are only valid for isothermal plasmas. Since many phases of the flare in the temperature range $1.10^{7}$ to $3.10^{7} \mathrm{~K}$ are close to 1sothermal, the diagnostic is a reasonable one, although of little use in determining the detailed departures from an isothermal situation.

In an attempt to generalise this analysis, some workers have tried to interpret the BCS 1 ine insensities in terms of a differential emission measure $N_{e}^{2} d V / d(\log T)$ vs $T$. Thus BelyDubau and Gabriel (unpublished) have developed a technfque based upon a Fourier deconvolution to fit such a function to the above eight eraission line intensities. However, it is well known that such an inversion is 111-conditioned when the width of the core functions (temperature dependence of each 1ine) is not much smaller than the range of line temperatures available. In this case, the width in $\log \mathrm{T}$ is 0.5 while the range in $\log \mathrm{T}$ is 0.9. Thus the precision obtainable with this narrow range of lines is poor. The deconvolution result showed clearly the changing temperature with time of the high 
temperature flare component. It was however of less use in determining the temperature width of this component, nor the existence of any high energy tail, thermal or non-thermal.

In general, the HXIS Instrument records six energy bands of x-ray data, covering $3.5 \mathrm{kV}$ to $30 \mathrm{kV}$. All bands include continuum. The lower bands have a significant line contribution in addition. The usual technique for interpreting these fluxes is to assume the calculated solar spectra of Mewe and Gronenschild /4/ together with the measured channel response, and interpret adjacent channel ratios in terms of temperature. Here again there is an implicit assumption of an 1sothermal plasma. Temperatures obtained fron the ratios of different palrs of channels are found to be different. Some of this was recently traced /5/ to an error in the atomic parameters of lines in Channel 2, but even after correction there are still discrepancies. These may be accounted for by departures from isothermal conditions. Yet another effect is perhaps due to a contribution in the harder channels of bremstrahlung due to electron streams. Analysis of flares using the channel ratio technique has reported high temperatures $\sim 5.10^{7} \mathrm{~K}$ over a large volume at the top of flare loops. Such temperatures appear to be inconsistent with BCS data, where they would be expected to produce strong Fe XXVI emission.

At the higher energy of the HXRBS channels, the emission in the impulsive phase is dominated by the bremsstrahlung from electron streams. Indeed it is usually assumed to be totally produced in this way. A further assumption is usually that the electrons responsible have a power-law spectrum. Channel ratios then give the spectral index, and their intensity gives the beam intensities. All of these assumptions are open to question. Some of the channels are sensitive to the thermal plasma emission. The power law, even if partly valid, must have a cut-off at low energy, and this cut-off also affects the channel responses. But there is no a priori reason to assume a power law.

Finally, whatever portion of the HXRBS signal can ultimately be attributed to electron beams gives the time-signature of the impulsive phase, which is of vital importance in deducing the energy release.

We can thus summarise the problem areas introduced by analysing the three instrument's data separately, and by some of the techniques employed:-

(a) limitations of isothermal assumption

(b) 111-conditioned inversion to obtain differential enission measures

(c) significant signals may be produced in a channel by large fluxes at energies well away from the channel peak

(d) uncertainties in electron beam energy distribution and cut-off.

\section{EMISSION MEASURE MODEL}

The present approach is to develop a generalised model based upon a differential emission measure and electron beam parameters, and to compute the absolute signals predicted in the 8 BCS, 6 HXIS and 15 HXRBS channe1s. For the thermal contribution, the count-rate in channel, $i$, can be expressed as

$$
C_{i}=A_{i} \int G_{i}(T) \cdot Y(T) \cdot d(\log T)
$$

where $Y(T)$ is the differential emission measure given by

$$
N_{e}^{2} \frac{d V}{d(t a g T)} \text {, }
$$

$G_{f}(T)$ is the temperature sensitivity of the channel $i$. In the case of BCS lines it is analagous to the Pottasch function $g(T)$, but can also be defined for HXIS and HXRBS channels to include many lines plus continuum, taking account of instrument window functions.

$A_{f}$ is a constant that includes instrument geometry, and overall sensitivity, element abundance, etc.

For electron beam excitation, the usual atomic theory is used for thin target excitation, whilst the formulation of Brown $/ 6 /$ has been adopted for thick target brensstrahlung.

No attempt has been made to invert the observed fluxes in order to derive a mathematically optimum model. It was considered impractical for inversion to take account of the variable accuracy of the observations; e.g. the satellite to resonance line ratio in one channel is better than 10\%, whereas comparison between instruments is perhaps $~ 30 \%$. The aim is rather to investigate the range and limits to models which can be made to fit the observed data 
set. Whilst it is possible to consider a generalised thermal differential emission measure without making any assumptions regarding geometry, the introduction of electron beams can require the use of additional parameters which do imply some such assumptions. The hot plasma DEM is defined in the usual way. The electron beam total current is defined and this together with an electron energy spectrum gives the thick-target bremsitrahlung. The electron energy spectrum is freely variable, being made up from the summation of monoenergetic components. We estimate also the comparatively weak contribution, which comes from the effect of the beam on the hot $\left(>10^{7} \mathrm{~K}\right)$ plasma. This effect is small or negligible compared with the other two components, but it is for this third component that restrictive assumptions must be made which depend on geometry (e.g. relative size of beam and hot plasma). Thus, overall, the effect of any implicit geometric assumptions is expected to be quite small.

The atomic data for excitation of the lines is that derived in the three references given above. However, for DEM analysis, ionization balance data is also required. Work by Antonucci et al. $17 /$, (and further unpublished work) has used a study of the flare data from the BCS, Hinotori and $\mathrm{P} 78-1$ in order to derive ratios of Li-like to He-like lons in Ca and $\mathrm{Fe}$ as a function of temperature. The ionization balance calculations of Jacobs et al. /8,9/ have been modified to take account of this work, and then used in the present modelling.

\section{FLARE OF 1980 JUNE $29,18.22$ UT}

As a first attempt the flare of 29 June 1980 , 18.23 hrs has been chosen for analysis. Time histories of some of the channels used are shown in Figure 1. An attempt was made to derive models which reproduce the observations for a number of times throughout this flare. It is not surprising to find that in general it is possible to fit more than one model to the data. Uniqueness can only be forced by making restrictive assumptions. Thus, analogous to the maximum entropy formulation in the autonatic inversion techniques, one can look for the simplest fit based upon one or two smooth maxima in the differential emission measure.

Attempts to fit two times during the flare, during the impulsive phase, and at the peak of the gradual phase, are shown in Figure 2 . Figure 2 a) shows the DEM's adopted, whilst Figure 2 b) shows the ratios of calculated to measured signals. For the later gradual phase, attempts to fit an all thermal DEM with no beams can be quite successful. However, even at this stage, a second maximum or hump in the DEM is found at around $10^{8} \mathrm{~K}$. During the earlier impulsive phase, it is impossible to obtain a good fit from a purely thermal DEM. The systematic deviations found can only be matched by assuming also that a power law electron beam dissipates its energy in thick target emission. Note

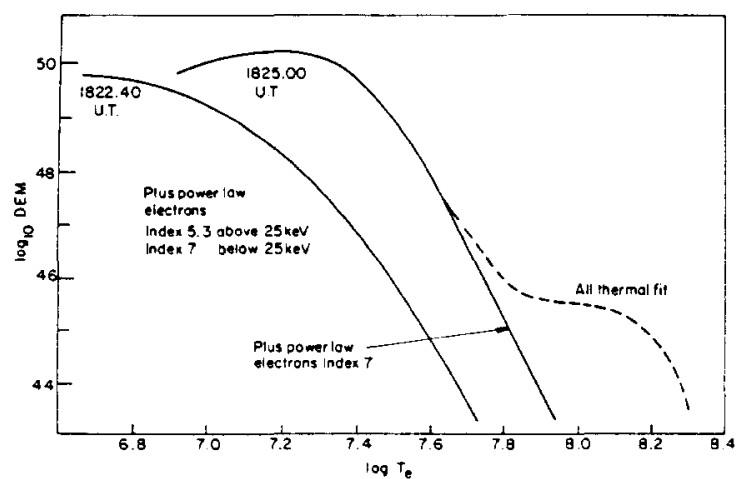

(a)

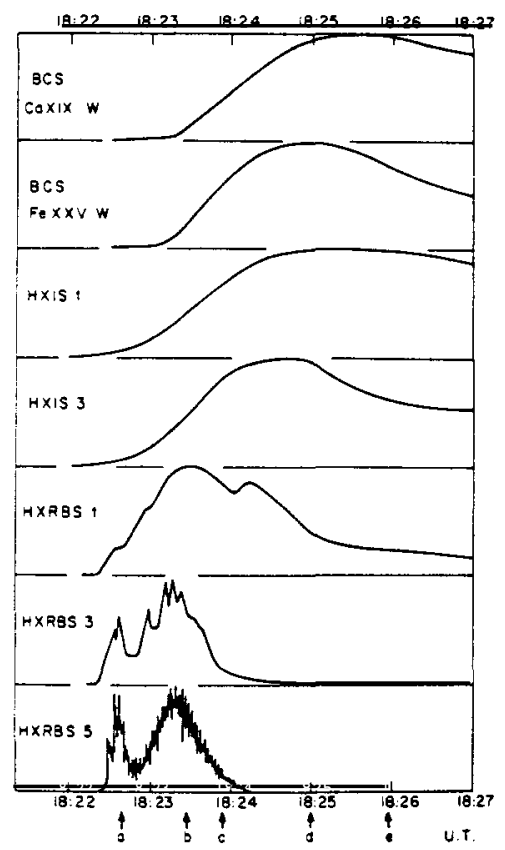

Fig. 1. Time development of the flare of 1980 June 29, $1822 \mathrm{UT}$, showing some of the channels used.

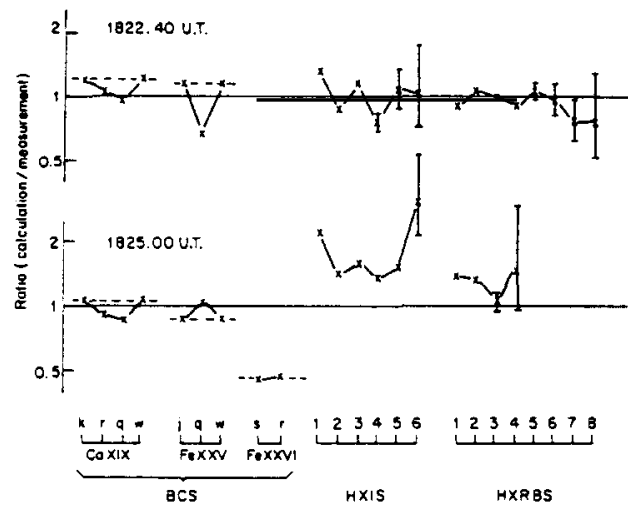

(b)

Fig. 2. Derived differential emission measure (a) and a comparison of calculated and observed count rates (b) for times during the impulsive phase and at the peak of the gradual phase. 
especially the quality of the fit shown in Figure 2 b). Whilst errors approaching factors of 2 have been permitted between the BCS Fe XXV and Fe XXVI channels, the dielectronic satellite ratios $\mathrm{Ca} X I X(k / w), F e \operatorname{XXV}(j / w)$ and the Fe XXVI $(s / r)$ have been required to be consistent, i.e. to lie on horizontal lines in this plot. Similarly, the "W" shape formed by the first 5 HXIS channels has been accepted only because it occurs consistently in all fittings, and must therefore represent a channel or atomic parameter calibration error. Note especially the high observed value for Fe XXV $q$ in the impulsive phase. This can be attributed to a departure from ionization equilibrium giving a high Fe XXIV concentration at this time. Since this departure lasts for less than $30 \mathrm{~s}$, we can deduce using the ionization rate for Fe XXIV that the flare electron density is in excess of $210^{10} \mathrm{~cm}^{-3}$ at this time.

It is of interest also to plot the values of the integrand of equation 1),

$$
G_{i}(T) . Y(T)
$$

for a number of lines, since this shows the actual temperatures at which those lines are formed within the real flare. For this purpose we have used the gradual phase fit to a pure therma 1 DEM. The result is shown in Figure 3. Although the DEM has a maximum at $\log$ $T=7.16$ and a full $\frac{1}{2}$ width of only $\Delta \log T=$ 0.15 , each of the lines is formed at a different temperature. Also shown on Figure 3 , by means of the arrows are the temperatures that would be determined from the satellite line ratios if one assumes an isothermal plasma. This ranges from $1610^{6} \mathrm{~K}$ for Ca XIX to $2710^{6} \mathrm{~K}$ for Fe XXVI. Clearly the full DEM is a much more satisfactory description of the plasma properties.

For further work, we make the following assumption in the fitting. A single smooth peak in DEM is adopted, of a shape to provide the best fit to all the BCS line spectra, for a given time in the flare. The missing high energy component is then provided using power law electron distributions and thick target emission. A single power law is used where possible, but it is sometimes necessary to use the sum of two. In all cases a cut-off of $10 \mathrm{keV}$ is used. Increasing this to 15 $\mathrm{keV}$ has only a minor effect on the fitting. The results are shown in Figure 4 as a function of time. The total thermal emission

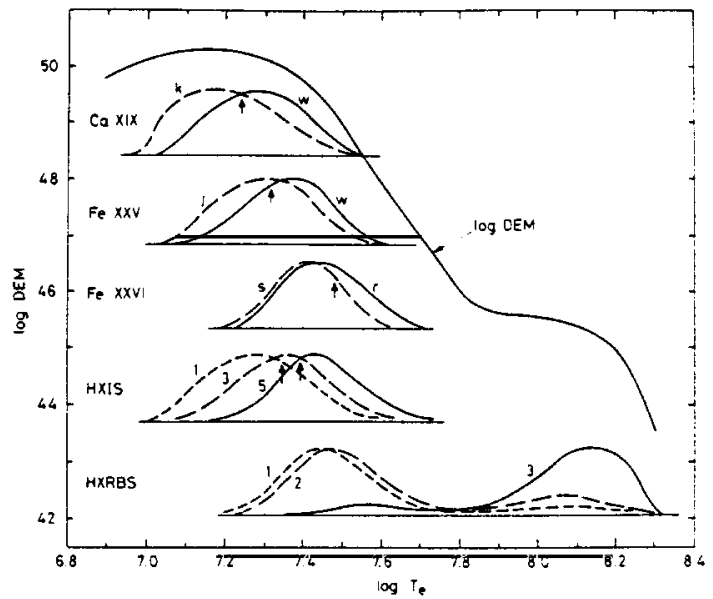

Fig. 3. Contributions as a function of temperature from several channels, calculated for the "thermal" phase, from the modelling of Figure 2.

measure (above $\log T=6.7$ ) is shown in the upper curve together with the peak temperature. These show the characteristic previously-observed variation with time. The beam component is always required. In the later gradual phase it has an electron index of around 7 and decays with time. During the earlier impulsive phase, it is necessary to introduce a second component with a harder index around 5.3 which follows in time the characteristic impulsive time profile of the flare.

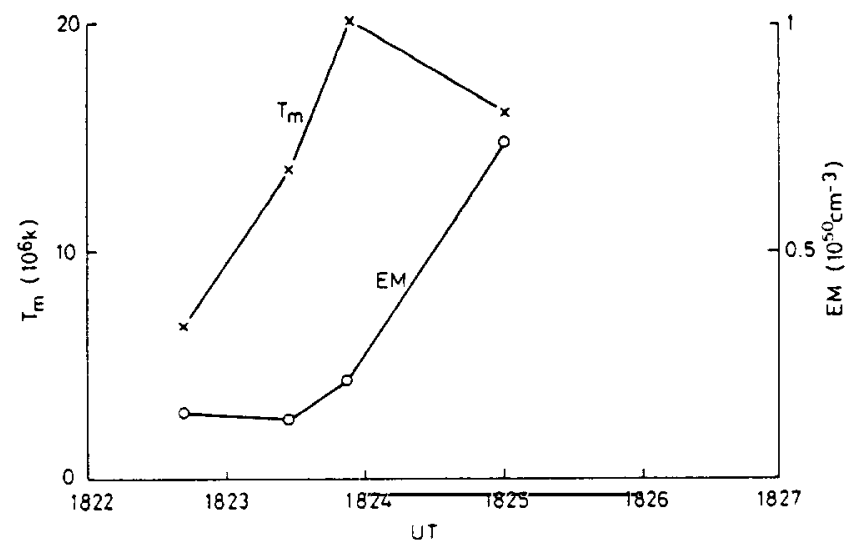

(a)

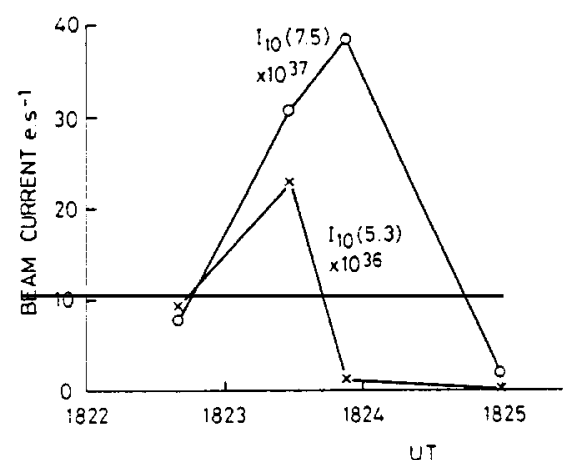

(b)

Fig. 4. The variation with time of the thermal component (a) and the beam component from the present analysis. 
CONCLUSION

By fitting the observations from three x-ray instruments on SMM, it 1s possible to gain improved understanding of the physical properties of the flare plasma. Whilst the fittings are not unique, it is possible to obtain consistent and credible results on the assumption of minimising the complexity of the models fitted. An attempt has been made to interpret the flare of 1980 June 29, 18.22 UT in this manner. A consistent fit is obtained on the basis of a single nearly isothermal component, plus thick target bremsstrahlung from power law electrons. Hard electrons with an index of 5.3 are found to colncide with the impulsive phase and could be associated with the primary energy release. The thermal emission measure is consistent with loops being filled with evaporated chromospheric plasma and establishlng a temperature and temperature gradient through conductive and radiative cooling. The remalning softer electron beam (index 7) persists well into the gradual phase of the flare and 1 ts origin is not well understood.

It will be important to apply this analysis to other flares in order to understand the extent to which these characteristics are typical, or special to this one.

\section{ACKNOWLEDGEMENTS}

The X-Ray Polychromator has been built by a consortium from Lockheed Palo Alto Research Laboratorles, The Mullard Space Sclence Laboratory and the Rutherford Appleton Laboratory. The Hard X-Ray Imaging Spectrometer was built by the Space Research Laboratory Utrecht and the University of Birmingham team. The Hard X-Ray Burst Spectrometer was provided by Goddard Space Flight Center. This work was stimulated by the NASA and UK SMM Workshops in $1983 / 4$.

\section{REFERENCES}

1. F. Bely-Dubau, J. Dubau, P. Faucher and A.H. Gabriel, Mon. Not. Astron. Soc., 198, 239, $(1982 \mathrm{a})$.

2. F. Bely-Dubau, J. Dubau, P. Faucher, A.H. Gabriel, M. Loulergue, L. Steenman-Clark, S. Volonte, E. Antonucct and C.G. Rapley, Mon. Not. R. Astron. Soc., 201,1155 (1982b).

3. J. Dubau, A.H. Gabriel, M. Loulergue, L. Steenman-Clark and S. Volonté, Mon. Not. R. Astron. Soc., 195, 705, (1981).

4. R. Mewe and E.H.B.M. Gronensch11d, Astron. and Astrophysics Suppl. 45, 11, (1981).

5. R. Mewe, E.H.B.M. Gronenschild and van den Oord, Astron. and Astrophys. (to be published).

6. J. Brown, Solar Phys., 18, 489-502, (1971).

7. E. Antonucc1, A.H. Gabriel, G. Doyle, J. Dubau, C. Jordan and N. Veck, Astronomy and Astrophysics 133, 239, (1984).

8. V.L. Jacobs, J. Davis, P.C. Kepple and M. Blaha, Ap.J., 211, 605, (1977).

9. V.L. Jacobs, J. Davis, J.E. Robertson, M. Blaha, J. Ca1n and M. Davis, Ap.J., 239, $1119,(1980)$. 